\author{
Asian Journal of \\ Medical and Biological Research \\ ISSN 2411-4472 (Print) 2412-5571 (Online) \\ www.ebupress.com/journal/ajmbr
}

\title{
Article \\ Clinical presentation and treatment in patients with acute coronary syndrome in a tertiary care general hospital in Bangladesh
}

Monalisa Monwar ${ }^{1}$, Ambia Khatun ${ }^{2}$, Masud Parvez ${ }^{3}$, Tarannum $\mathrm{Naz}^{4}$, Mir Imam Ibne Wahed ${ }^{5 *}$, Md. Ekramul Haque $^{6}$, Sumon Chandra Debnath ${ }^{7}$ and Md. Imran Khan ${ }^{8}$

${ }^{1}$ Department of Pharmacy, Varendra University, Rajshahi, Bangladesh

${ }^{2}$ Department of Pharmacy, University of Rajshahi, Rajshahi, Bangladesh

${ }^{3}$ Department of Pharmacy, Varendra University, Rajshahi, Bangladesh

${ }^{4}$ Department of Pharmacy, University of Rajshahi, Rajshahi, Bangladesh

${ }^{5}$ Department of Pharmacy, University of Rajshahi, Rajshahi, Bangladesh

${ }^{6}$ Communicable Disease Control (CDC), DGHS, Mohakhali, Dhaka, Bangladesh

${ }^{7}$ HCMP, BRAC, Bangladesh

${ }^{8}$ Department of Biochemistry, Rajshahi Medical College, Bangladesh

*Corresponding author: Dr. Mir Imam Ibne Wahed, Professor, Department of Pharmacy, University of Rajshahi, Rajshahi 6205, Bangladesh. E-mail: wahed_mir@ru.ac.bd

Received: 07 March 2018/Accepted: 18 March 2018/ Published: 29 March 2018

\begin{abstract}
Acute coronary syndrome (ACS) is a leading cause of death among people in Bangladesh. The aim of the study was to analyze the clinical course and treatment strategies in patients with ACS and to determine to what extent management of ACS in a tertiary care general hospital in Bangladesh adhered to current guidelines. This study was carried out in the coronary care unit (CCU) of a tertiary care general hospital, Rajshahi, for a period of 3-months. A total number of 240 patients presenting with ACS were included in our study and the most common symptoms were acute chest pain (90\%) and dyspnea (49\%). The study group comprised of $27 \%$ female and $73 \%$ male patients with varying risk factors including hypertension (45\%), hyperlipidemia (43\%), family records of coronary artery disease (CAD) (20\%), diabetes (17\%) and smoking (15\%). The most frequent ECG finding in patients was T wave change (71\%), pathological Q wave (67\%), ST segment elevation (33\%), ST depression (9\%), whereas, $12 \%$ patients with ACS reported to have normal ECG. Patients with ACS had elevated levels of SGOT and CK-MB. Troponin I level was positive in $100 \%$ of the patients with a mean peak troponin level of $1.5 \pm 0.15 \mathrm{ng} / \mathrm{ml}$. In clinical setting, the patients were immediately managed with isosorbidedinitrate (58\%), streptokinase (40\%) and intravenous heparin (LMWH) followed by (46\%) and (54\%) of aspirin and aspirin-clopidogrel combinations respectively. In addition, long-term management with antihypertensive included $\beta$-blocker (58\%), calcium channel blocker (29\%), ACE-I (25\%) and diuretics (12\%). Anti-diabetic (18\%) drugs were also prescribed in patients with co-existing diabetes and CAD. Adherence to guidelines is limited by lack of funds and resources in the hospital; however, attention must be paid to improve patient outcome. The average hospital stay ranges 2-3 days and hospital mortality was $10 \%$.
\end{abstract}

Keywords: acute coronary syndrome; patients; tertiary care general hospital

\section{Introduction}

Coronary artery disease (CAD) is one of the major cause of cardiovascular mortality among people worldwide (Islam and Majumder, 2013). According to the WHO, 17.3 million deaths were attributable to cardiovascular disease of which $42 \%$ died as a result of myocardial infarction (Mendis et al., 2011). It has been reported that angina due to ischemic heart disease (IHD) affects approximately 112 million people (NHFoA, 2007) and men are more likely to have an AMI then women and that the risk of AMI increases with age (Barnett et al., 2001). 
The pathology underlying the development of ACS results from the erosion and rupture of a fibrous cap containing a lipid-rich atherosclerotic plaque that precipitates thrombus formation within the coronary artery, and the critical consequence is downstream myocardial ischemia [add ref]. In Bangladesh, the CVD mortality rates increased by 30-fold among males and 47-fold in females during the period of 1986-2006 (Muhit et al., 2012). The prevalence of CAD is about 1.85-3.4\% in rural and 19.6\% in urban areas (Zaman et al., 2007; Ahsan et al., 2009; Saquib et al., 2011).

A total of 780 patients visited National Institute of Cardiovascular Diseases (NICVD), Dhaka have lipid disorder (47\%), hypertension (28\%), stroke (16\%), ischemic heart disease (22\%) heart failure (27\%) and myocardial infarction (24\%) (Karar et al., 2009).

Elevated levels of total cholesterol, levels of high-density lipoprotein (HDL) less than $40 \mathrm{mg} / \mathrm{dL}$, diabetes and high blood pressure are associated with an increased risk of coronary atherosclerosis and MI (Vos et al., 2012; Regnante, 2009). Several other factors contributing to the development of MI include dyslipidemia, obesity, chronic kidney disease, psychosocial condition, smoking habit, excessive physical activity and use of drugs such as non-steroidal anti-inflammatory drugs (NSAIDs) and alcohol intake (Hung et al., 1995; Rutten, 2000).

The echocardiography (ECG) is usually helpful in confirming the diagnosis; however, it may be difficult to interpret if there is bundle branch block or evidence of previous MI. ECG is a very useful technique for assessing both ventricular function and other complications such as mural thrombus, cardiac rupture, ventricular septal defect, mitral regurgitation and pericardial effusion. Other techniques include chest X-ray, measurement of serum creatine kinase (CK), troponin I level (Greenlund et al., 2006; Grzegorz, 2013). Creatine kinase-MB enzyme is much more cardiac specific than $\mathrm{CK}$ alone, and is useful for the early diagnosis of acute myocardial infarction (Nelson et al., 2001) typically detectable in the serum 4-6 hours after the onset of ischemia, peaks in 12-24 hours, and normalizes in 2-3 days. Recently, an elevated levels of troponin I is considered as the most useful and predictive markers in identifying patients with acute myocardial infarction (Barnett et al., 2001).

Emergency and long-term management strategies of AMI include administration of thrombolytic drugs, antiarrhythmic, anti-hypertensive drugs such as $\beta$-blockers (metoprolol, propranolol), calcium channel blockers (amlodipine), vasodilators (nitroglycerin) and anticoagulants (Akashi, 2008; Sayeed et al., 2010).

It is evident that CAD is major cause of death in present world and current guidelines are therefore, aimed at optimizing care and outcome of patients with acute coronary syndrome (Puleo et al., 1989).

In the acute phase ACS patients require rapid diagnosis and early reperfusion to minimize infarct size and to prevent complications. Several factors determine treatment delay with its major contributors being patient delay such as ignorance, unaffordable cost, physician delay such as unavailability; and also lack of adequate facilities and trained person in hospitals (Bock et al., 1999; Atary, 2010).

The aim of this study was to analyze the clinical presentation and treatment strategies in patients with ACS at hospital settings in a tertiary-care medical hospital in Bangladesh adhered to current guidelines.

\section{Materials and Methods}

\subsection{Study design and settings}

This cross-sectional study was carried out with an objective to assess the clinical presentation and treatment strategies in patients with ACS at hospital settings in a tertiary-care medical hospital in Bangladesh adhered to current guidelines. The study was conducted during the months October-December in 2012 at the CCU of Rajshahi Medical College, Bangladesh.

\subsection{Study population and sample size}

The study involved a total number of 240 patients hospitalized with the chief complaints of acute chest pain, palpitation, excessive sweating, respiratory distress, and apnea. Purposive sampling was done to find out the study population.

\subsection{Data collection process}

Data were collected through a semi-structured questionnaire that included admission/discharge date, clinical presentation, diagnostic tests, biochemical analysis, medication and outcomes during hospital stay. Only a few biochemical tests for serum glutamic oxaloacetic transaminase (SGOT), CK-MB and troponin I were advised within the hospital settings. Thus, the results SGOT, CK-MB and troponin I from 25 patients were collected from patients chart. 


\subsection{Statistical analysis}

Data was cleaned for completeness and consistencies, coded and entered in to SPSS version 20 for analysis. The results were organized, summarized and presented using appropriate descriptive measures such as text, tables, frequencies and percentage.

\section{Results}

During the study period, 240 patients were admitted at the CCU, Rajshahi Medical College Hospital, Bangladesh. Baseline characteristics of the patients are presented in Table 1 . The study included $27 \%$ female and 73\% male patients. The age of patients commonly encountering ACS ranges from 45-54 years, whereby the mean age of patient was $50 \pm 4$ years. The five most common risk factors were hypertension (45\%), hyperlipidemia (43\%), a family history of CAD (20\%), diabetes (17\%) and smoking (15\%) (Table 1). Most of the patients had history of cardiac abnormalities including unstable angina (21\%), AMI (38\%), chronic MI (40\%) patients and also reported to have aortic stenosis (11\%) which was unrelated to clinical presentation of ACS. The average hospital stay ranges 2-3 days and hospital mortality was $10 \%$ (Table 1).

The most of the patients were admitted with a chief complaints of chest pain in 216 (90\%), dyspnea in 118 (49\%), sweating in 55 (23\%), nausea-vomiting in 48 (20\%) and ankle edema in 24 patients (10\%) (Table 2).

The characteristic changes in ECG in patients with ACS given in Table 2. The contemporary trend in monitoring the ECG changes allows distinguishing patients with acute coronary syndrome (those with unstable angina and those with $\mathrm{Q}$ wave infarction) on the basis of $\mathrm{T}$ wave inversion or ST segment depression or elevation or both. All the patients had elevated levels of SGOT, CK-MB and troponin (Table 3).

Medications used during the period of hospitalization are listed in Table 4. The majority of the patients were immediately managed by isosorbidedinitrate, streptokinase, aspirin, heparin, aspirin-clopidegrol combinations and statin during the course of hospital stay. All the patients taking either aspirin or statin alone or aspirinclopidegrol combinations. In addition to anti-thrombotic and lipid lowering drugs long-term management with antihypertensive drugs, $\beta$-blocker, CCBs, ACE-I and diuretics. In addition anti-diabetic drugs were also prescribed in patients with diabetes and CAD. After discharge $\beta$-blocker and ACE-I were continued in most of patients in addition with aspirin, clopidogrel and statin. Patients with edema suggested to have diuretics and a few with unstable angina were requested to carry nitroglycerin spray. The patients were usually advised to take rest for couple of weeks, do not eat egg, butter, ghee, egg yolk, meat, etc and cigarette, tobacco, drugs of abuse and avoid extra salt. Finally, patients were requested to visit cardiologists if any further problem arises. Followup data were not available as the study conducted for short-term period. Patient died of cardiogenic shock were excluded from the study. The average hospital stay was 2-3 days and hospital mortality was $10 \%$ (Table 4).

Table 1. Baseline socio-demographic characteristics of patients $(n=240)$.

\begin{tabular}{ll}
\hline Characteristics & Patients (\%) \\
\hline Sex & $176(73 \%)$ \\
Male n (\%) & $64(27 \%)$ \\
Female n (\%) & $50 \pm 13$ \\
Age $($ Mean \pm SD) & $28(12 \%)$ \\
$<45$ n (\%) & $86(36 \%)$ \\
$45-54$ n (\%) & $80(33 \%)$ \\
$55-64$ n (\%) & $46(19 \%)$ \\
$>64$ n (\%) & \\
Risk factors $\boldsymbol{n}(\%)$ & $108(45 \%)$ \\
Hypertension & $104(43 \%)$ \\
Hyperlipidemia & $48(20 \%)$ \\
Family history of CAD & $40(17 \%)$ \\
Diabetes & $38(15 \%)$ \\
Smoking & \\
Medical History of cardiac abnormalities $n(\%)$ & $26(11 \%)$ \\
Aortic stenosis & $52(21 \%)$ \\
Unstable angina & $92(38 \%)$ \\
Acute MI & $96(40 \%)$ \\
Chronic MI & \\
\hline
\end{tabular}


Table 2. Clinical presentation along with ECG and cardiac biomarkers in patients with acute coronary syndrome (ACS).

\begin{tabular}{ll}
\hline Clinical Presentation & Patients (\%) \\
\hline Chief Complaints & $216(90 \%)$ \\
Chest pain, $\mathrm{n}(\%)$ & $118(49 \%)$ \\
Dyspneoa, n (\%) & $48(20 \%)$ \\
Vomiting, n (\%) & $55(23 \%)$ \\
Sweating, $\mathrm{n}(\%)$ & $24(10 \%)$ \\
Ankle edema, $\mathrm{n}(\%)$ & \\
\hline
\end{tabular}

Table 3. ECG and cardiac biomarkers in patients with ACS.

\begin{tabular}{ll}
\hline Pathological changes & Mean $\mathbf{( \% )}$ \\
\hline ECG changes & \\
ST elevation n (\%) & $78(33 \%)$ \\
T wave change n (\%) & $170(71 \%)$ \\
Pathological Q wave n (\%) & $162(67 \%)$ \\
ST depression n (\%) & $22(9 \%)$ \\
Without change n (\%) & $30(12 \%)$ \\
Biomarker & \\
SGOT (mg/dl) & $120 \pm 30$ \\
CK-MB (IU/L) & $316 \pm 80$ \\
Troponin I (mg/ml) & $1.57 \pm 0.15$ \\
\hline
\end{tabular}

Table 4. Treatment strategies in patients with ACS during hospital stay and discharge.

\begin{tabular}{ll}
\hline Drug n (\%) & Patients (\%) \\
\hline Streptokinase & $96(40 \%)$ \\
Isosorbidedinitrate & $140(58 \%)$ \\
Heparin & $32(13 \%)$ \\
Aspirin & $110(46 \%)$ \\
Aspirin + Clopidegrol & $130(54 \%)$ \\
Statin & $104(43 \%)$ \\
Proton Pump Inhibitor & $240(100 \%)$ \\
Calcium Channel Blocker & $70(29 \%)$ \\
$\beta$-blocker & $140(58 \%)$ \\
Diuretics & $30(12 \%)$ \\
ACE-I & $60(25 \%)$ \\
Anti-diabetic & $40(18 \%)$ \\
\hline
\end{tabular}

\section{Discussion}

This survey represents a recent clinical study on ACS report in the Bangladeshi population. The study involved a total number of 240 patients hospitalized during the 3 -months period. The common symptoms were acute chest pain (90\%), dyspnea (49\%), sweating (23\%), vomiting (20\%) and ankle edema (10\%). Our results were in accordance with findings of Regnante, 2009. We noticed a low rate of vomiting and nausea symptoms that were usual in other studies on ACS. Our study group comprised of $27 \%$ female and $73 \%$ male patients with numerous cardiovascular risk factors including hypertension (45\%), hyperlipidemia (43\%), family records of CAD (20\%), diabetes (17\%) and smoker (15\%). Bock et al., 1999 reported the similar risk factors that can predispose to coronary artery disease, except the variability of occurrence.

Most of the patients (89\%) had history of ACS and the remaining had aortic stenosis (11\%).In the present study, ECG was performed in all patients irrespective of whether physician had provisionally diagnosed ACS or not (Zaman et al., 2007). Although ECG findings are of specific markers of ACS, the ECG chows low sensitivity and is of limited value for ACS diagnosis. The most frequent finding was T wave change noticed in $71 \%$ patients with ACS, followed by pathological Q wave in 67\%, ST segment elevation in 33\% and ST depression in $9 \%$ patient. ST-Segment elevation in precordial leads present on admission has been observed by M Islam and A Majumder, 2013 and later on there might be the appearance of pathological Q wave Saquib et al., 2011. 
Unlike the Ahsan et al., 2009 in which 3.3\% of the cases had no ECG abnormalities, our study reports only $12 \%$ of the cases with normal ECG.

All the patients had elevated levels of SGOT, CK-MB and troponin I. The average value of SGOT was $120 \pm 30$ $\mathrm{mg} / \mathrm{dl}$, CK-MB was $316 \pm 80 \mathrm{U} / \mathrm{L}$ and troponin was $1.57 \pm 0.15 \mathrm{mg} / \mathrm{ml}$, and all the values were consistent with the patient disease outcome. Troponin I level was positive in $100 \%$ of the patients with a mean peak troponin level of $1.57 \mathrm{mg} / \mathrm{ml} \mathrm{compared} \mathrm{with} 2.7 \mathrm{mg} / \mathrm{ml}$ by Muhit et al., and 2012 and $6.9 \mathrm{mg} / \mathrm{ml}$ reported by Sayeed et al., 2010.

The majority of the patients were immediately managed by isosorbidedinitrate (58\%), streptokinase (40\%), aspirin (46\%), heparin (13\%), aspirin-clopidogrel combinations $(54 \%)$ and statin $(43 \%)$ during the course of hospital stay. Because of the clinical presentation and ECG abnormalities, most patients were initially treated for myocardial ischemia with loading doses of dual antiplatelet drugs (aspirin-clopidogrel) and intravenous heparin. There is no clear consensus on the management of patients with ACS, but most of our patients continued to receive Aspirin (ASA), $\beta$-blocker and ACE inhibitors. In addition to anti-thrombotic and lipid lowering drugs long-term management include the four most common antihypertensive drugs, $\beta$-blocker (58\%), calcium channel blocker (29\%), ACE-I (25\%) and diuretics (12\%). Anti-diabetic (18\%) drugs were prescribed in patients with diabetes and coronary artery disease (Vos et al., 2012; NHFoA, 2007). When medical treatment fails the patients are advised to undergo coronary angiography. Our study revealed that only a few patient (1 patient in week) suggested to undergone angiography followed by angioplasty. There were no reports of percutaneous coronary intervention (PCI).

Most of the patients (89\%) had history of ACS admitted to CCU of tertiary care general hospital. Clinical presentation is indistinguishable from ACS, but the course is milder and the outcomes were better. Adherence to guidelines is limited because of the absence of physicians, skilled manpower, technician and also lacking of funds, no laboratory facilities and limited resources in the hospital setting; however, attention must be paid to improve services in CCU to properly manage the ACS and to save lives.

Our study has several limitations, such as, the data presented here is based on retrospective analysis. Secondly, the number of patients was relatively small. Thirdly, in very few cases laboratory parameters were advised. Fourthly, no follow-up data were obtained due to time limit of the study and was conducted for only three months, from October to December and this data may slightly vary from the scenario other months of the year.

\section{Conclusions}

The most common symptoms of patients were acute chest pain and dyspnea with varying duration and risk factors including hypertension, hyperlipidemia, family records of coronary artery disease (CAD), diabetes and smoking. The most frequent ECG finding in patients was T wave change, pathological Q wave, ST segment elevation, ST depression, whereas, patients with ACS reported to have normal ECG. Most of the patients were immediately managed by isosorbidedinitrate, streptokinase, aspirin, heparin, and aspirin-clopidogrel combinations and statin during the course of hospital stay. Constancy to standard guidelines is inadequate to properly manage the patients with ACS in a district level tertiary care hospital in Bangladesh due to patient delay and poor management facilities in hospital settings. Therefore adequate attention will be provided from patient and hospital level to overcome the uncertain attack of ACS. Furthermore the government or policy maker should give more attention for improving the management and treatment facilities in government hospital and allocating a big budget in this sector to well management of such type of leading pandemic cause of death.

\section{Acknowledgement}

We are grateful to the hospital authority for the permission to conduct the study. We would like to thank all the study participants who added an extensive contribution during data collection.

\section{Conflict of interest}

None to declare.

\section{References}

Muhit A, O Rahman, SZ Raihan, M Asaduzzaman, MA Akbar, N Sharmin and ABM Faroque, 2012. Cardiovascular disease prevalence and prescription patterns at a tertiary level hospital in Bangladesh. Journal of Applied Pharmaceutical Science, 23: 80-84.

Nelson DE, D Holtzman, J Bolen, CA Stanwyck and KA Mack, 2001. Reliability and validity of measures from the Behavioral Risk Factor Surveillance System. Soz Praventivmed, 46: 3-42. 
Karras DJ and DL Kane, 2001. Serum markers in the emergency department diagnosis of acute myocardial infarction. Emerg. Med. Clin. North Am., 19: 321-337.

Barnett E, ML Casper and JA Halverson, 2001. Men and heart disease: an atlas of racial and ethnic disparities in mortality. First edition. Morgantown, WV: Office for Social Environment and Health Research, West Virginia University, 2001.

Rutten FH, 2000. Electrocardiography in primary care, is it useful. Int. J. Cardiol., 20: 199-205.

JL Bock, GX Brogan, CF McCuskey, HC Thode, JE Hollander and T Gunther, 1999. Evaluation of CK-MB isoform analysis for early diagnosis of myocardial infarction. J. Emerg. Med., 17: 75-79.

Atary JZ, 2010. Standardised pre-hospital care of acute myocardial infarction patients. Ntherlands Heart Journal, 18: 408-415.

Hung J, JYT Lam, L Lacoste and G Letchacovski, 1995. Cigarette smoking acutely increases platelet thrombus formation in patients with coronary artery disease taking aspirin. Circulation, 92: 2432-2436.

Greenlund KJ, WH Giles and NL Keenan, 2006. Heart disease and stroke mortality in the 20th century. The history and practice of public health in twentieth century America. Oxford, England, Oxford University Press, 2006.

Islam M and A Majumder, 2013. Coronary artery disease in Bangladesh: A review. Indian Heart Journal, 65: 424-435.

Sayeed MA, H Mahtab and S Sayeed, 2010. Prevalence and risk factors of coronary heart disease in a rural population of Bangladesh. Ibrahim Med. Coll. J., 4: 37-43.

Zaman MM, J Ahmed and SR Choudhury, 2007. Prevalence of ischemic heart disease in a rural population of Bangladesh. Indian Heart J., 59: 239-241.

National Heart Foundation of Australia (NHFoA), 2007. The burden of cardiovascular disease in Australia for the year 2003. Report by Vos T and Begg S, Centre for Burden of Disease and Cost effectiveness, University of Queensland School of Population Health.

Saquib N, J Saquib and T Ahmed, 2011. Cardiovascular diseases and type 2 diabetes in Bangladesh: a systematic review and meta-analysis of studies between 1995 and 2010. BMC Public Health, 12: 434-439.

Grzegorz O, 2013. Clinical presentation, treatment and long term out comes in patients with takotsubo cardiomyopathy. Pol. Arch. Med. Wewn., 6: 231-236.

Puleo PR, PA Guadagno, R Roberts and MB Perryman, 1989. Sensitive, rapid assay of subforms of creatine kinase MB in plasma. Clin. Chem., 35: 1452-1455.

Regnante R, 2009. Clinical characteristics and four year outcomes of patients in the Rhode IslandsTakotsubo Cardiomyopathy Registry. Am. J. Cardiol., 103: 1015-1019.

Ahsan S, KMHS Haque and M Salman, 2009. Detection of ischaemic heart disease with risk factors in different categories of employees of University Grants Commission. Univ. Heart J., 5: 20-23.

S Mendis, P Puska and B Norrving, 2011. Global Atlas on Cardiovascular Disease Prevention and Control. World Health Organization, Geneva 2011.

Vos T, AD Flaxman, M Naghavi, R Lozano, C Michaud and M Ezzati, 2012. Years lived with disability (YLDs) for 1160 sequelae of 289 diseases and injuries 1990-2010. Lancet, 380: 2163-2196.

Akashi YJ, 2008. Takotsubo cardiomyopathy. A new form of acute, reversible heart failure. Circulation, 118: 2754-2762.

Karar ZA, N Alam and KP Streatfield, 2009. Epidemiological transition in rural Bangladesh, 1986-2006. Glob Health Action, 2: 23-29. 